\title{
Changes of Biomechanical Characteristics of the Bone in Experimental Tibial Osteotomy Model in the Dog
}

\author{
Yasushi HARA $^{1)}$, Toshiyuki NAKAMURA ${ }^{2)}$, Hiroshi FUKUDA ${ }^{3)}$, Yasuji HARADA ${ }^{4)}$, Yoshinori NEZU ${ }^{1)}$ and \\ Masahiro TAGAWA ${ }^{1)}$ \\ ${ }^{1)}$ Division of Veterinary Surgery, School of Veterinary Medicine, Nippon Veterinary and Animal Science University, 1-7-1 Kyonan-cho, \\ Musashino-shi, Tokyo 180-8602, 2)Development Research Laboratories, Kaken Pharmaceutical, Co., Ltd., 301 Gensuke, Fujieda-shi, \\ Shizuoka 426-8646, ${ }^{31}$ Department of Materials Science and Technology, Science University of Tokyo, 2641 Yamazaki-cho, Noda-shi, \\ Chiba 278-0022 and ${ }^{4}$ Institute for Frontier Medical Sciences, Kyoto University, 53 Kawahara-cho, Shogoin, Sakyo-ku, Kyoto 606-8397, \\ Japan
}

(Received 21 January 2002/Accepted 17 September 2002)

ABSTRACT. Experimental osteotomy model of canine tibia was prepared to investigate the changes in biomechanical characteristics during the healing process. After 16 weeks, although radiographs revealed that fracture healing proceeded, the recovery rates of the $\mathrm{m}$ echanical parameters ranged from 12.3 to $47.3 \%$, compared to the intact side. After 32 weeks, those recovered to 68.9-93.2\%. These result s suggested that the biomechanical characteristics of the healing bone could not be recovered sufficiently even after the passage of the healing period which has been empirically proposed from clinical findings. It was also considered that evaluation of the mechanical par ameters using this osteotomy model would be suitable for investigating the effect of osteoinductive growth factors on fracture healing.

KEY WORDS: biomechanics, canine, osteotomy model.

J. Vet. Med. Sci. 65(1): 103-107, 2003

In the field of small animal orthopedics, a number of studies have been undertaken on surgical techniques for fracture reduction and fixation, local biological reactions at the fracture site, and healing mechanism, so far $[7,8,13$, 14]. The healing process of the diaphyseal fracture of a long bone after treatment with internal fixation, and the timing of implant removal are clinically estimated by the clinical function of the affected limb, including the degree of grounding and weight-bearing, and from the radiographic finding of the fractured bone [3,13]. However, of numerous studies reported previously, biomechanical approach interpreting bone tissue as a supporter has been rarely attempted $[2,11,16]$. The major function of long bones in the leg is to support to resist physiological stress imposed by gravity or muscle activities in the body and nonphysiological stress imposed by external force such as traffic accident or fall. Therefore, restoration of normal biomechanical properties during the healing process should be interpreted as true healing. In the present study, diaphyseal osteotomy of the tibia was experimentally produced in the dog and was fixed by intramedullary pinning. We evaluated the changes in biomechanical characteristics of the healing bone after osteotomy.

Twenty-one clinically healthy Beagle dogs, which ranged from 11 to 15 months (mean 12.1 months) in age, and from 6.5 to $11.0 \mathrm{~kg}$ (mean $8.9 \mathrm{~kg}$ ) in body weight respectively, were used. They consisted of 8 males and 13 females. Under general anesthesia with isoflurane (Abbott Laboratories, Abbott Park, IL, U.S.A.) and oxygen, the right hindleg was disinfected and transversal osteotomy was made in the middle of the tibial diaphysis using an oscillating bone saw (Striker, Kalamazoo, MI, U.S.A.). Then an intramedullary pin (3 $\mathrm{mm}$ in diameter, Mathys, Bettlach, Switzerland ) was inserted and fixed along enough to cover the entire length of the medullary cavity of the tibia, and the wound was closed by a routine procedure. Perioperative analgesic management was done by the pre- and post-operative administration of butorphanol $(0.2 \mathrm{mg} / \mathrm{kg}$, iv). Butorphanol was administered for 3 days after surgery twice a day in the same dose. For 14 days after surgery, $30 \mathrm{mg} / \mathrm{kg}$ of ampicillin was orally administrated twice a day. External coaptation was applied with a hardening bandage (3M Health Care, St. Paul, MN, U.S.A.) for four weeks after surgery. The dogs were divided at random by the length of observational period into five groups ; observation at 2, 4, 8, 16, and 32 weeks after surgery. The groups assigned for observation at 2, 4, and 8 weeks consisted of $3 \mathrm{dogs}$ each. Those assigned for observation at 16 and 32 weeks consisted of 6 each. At the end of the observational period, the dogs were sacrificed by administrating an overdose of pentobarbital sodium. Then, the bilateral tibial bones were harvested, freed of surrounding soft tissue. After removal of the intramedullary pin, they were used for the measurement of biomechanical parameters. The surgical treatment and postsurgical management of the animals were carried out according to the Gudelines for the Care and Use of Laboratory Animals of Nippon Veterinary and Animal Science University. Radiographs were taken in the lateral and anterioposterior projections, before surgical treatment and immediately, 2, 4, 8, 16, and 32 weeks after surgical treatment. The bone mineral content (BMC) of the collected samples was measured by dual energy X-ray absorptiometry utilizing bone mineral analyzer (Aloka Co., Tokyo, Japan). For the measurement, a zone $25 \mathrm{~mm}$ proximal and $25 \mathrm{~mm}$ distal to the center of the fracture line was chosen as a region of interest for the surgical treated tibia. For the contralateral intact tibia, BMC was 
also measured at the corresponding region of interest [6]. Biomechanical properties of these samples were also examined by three-point bending test on all bone samples collected from both sides using universal material testing machine (Shimadzu Co., Kyoto, Japan). Load was applied on to the center of the tibial diaphysis from the anterior side of the tibia until break. The maximum load (ML) and deflection (D) were determined on the basis of the stressdeflection curve recorded in the test. In three-point bending test, the crosshead speed and the distance of two supporting points (L) in the metaphyses were established $5 \mathrm{~mm} / \mathrm{min}$ and $60 \mathrm{~mm}$, respectively. To measure the transversal area of the tibia, X-ray CT scanning (Yokogawa Medical Co., Tokyo, Japan) was performed at slice intervals of $2 \mathrm{~mm}$ on the region of fracture line using the tibial samples collected at each observational period. Based on the CT images obtained, the radius of bone (BR) and bone marrow cavity (BMR) were measured with window width and window level at 1,500 and 300, respectively. On the basis of these primary parameters, the following parameters were calculated basically by the previously reported method $[1,15$, 16].

Maximum bending strength $(M B S)=M L \times L \times B R / 4 I$

Flexural rigidity $(F R)=M L \times L^{3} / 48 D$

Young's modulus $(Y M)=M L \times L^{3} / 48 I D$

where $I=$ area moment of inertia.

$I=\pi\left(B R^{4}-B M R^{4}\right) / 4$

For the standardization of measured values among indi- vidual animals, BMC, ML, MBS, FR, and YM on the surgically treated side were expressed in terms of the rates of recovery or change, namely, the ratios of values on the surgically treated side to those on the intact side. For each parameter, the recovery rates at $2,4,8,16$, and 32 weeks were compared by one-way analysis of variance. Scheffe's comparison procedure was used to test for significant differences between groups at an experimentwise error rate of $\mathrm{p}<0.05$.

Figure 1 shows the lateral radiographs of the tibia in healing process after surgery. At 4 weeks, the periosteal callus was formed extensively near the osteotomized ends. At 8 weeks, the formation of external callus was completed and ossification at the osteotomized site became clearer. At 16 weeks, the remodeling of external callus was progressed, and the fracture line was completely disappeared.

Figure 2 shows the changes in recovery rate of BMC with time. BMC tended to increase its recovery rate from 2 weeks after surgical treatment. Although the recovery rate was increased to $142.0-159.0 \%$ at 4-32 weeks, no significance was found. BMC varied in parallel to the formation of external callus as revealed by radiographs.

Table 1 shows the mechanical parameters measured in the three-point bending test. The recovery rates of them in healing process are summarized in Fig. 3. The recovery rate of ML was increased with time, showing $93.2 \%$ in recovery rate at 32 weeks. The recovery rates at 32 weeks was significantly higher compared to those at $2-16$ weeks $(\mathrm{p}<0.001)$.

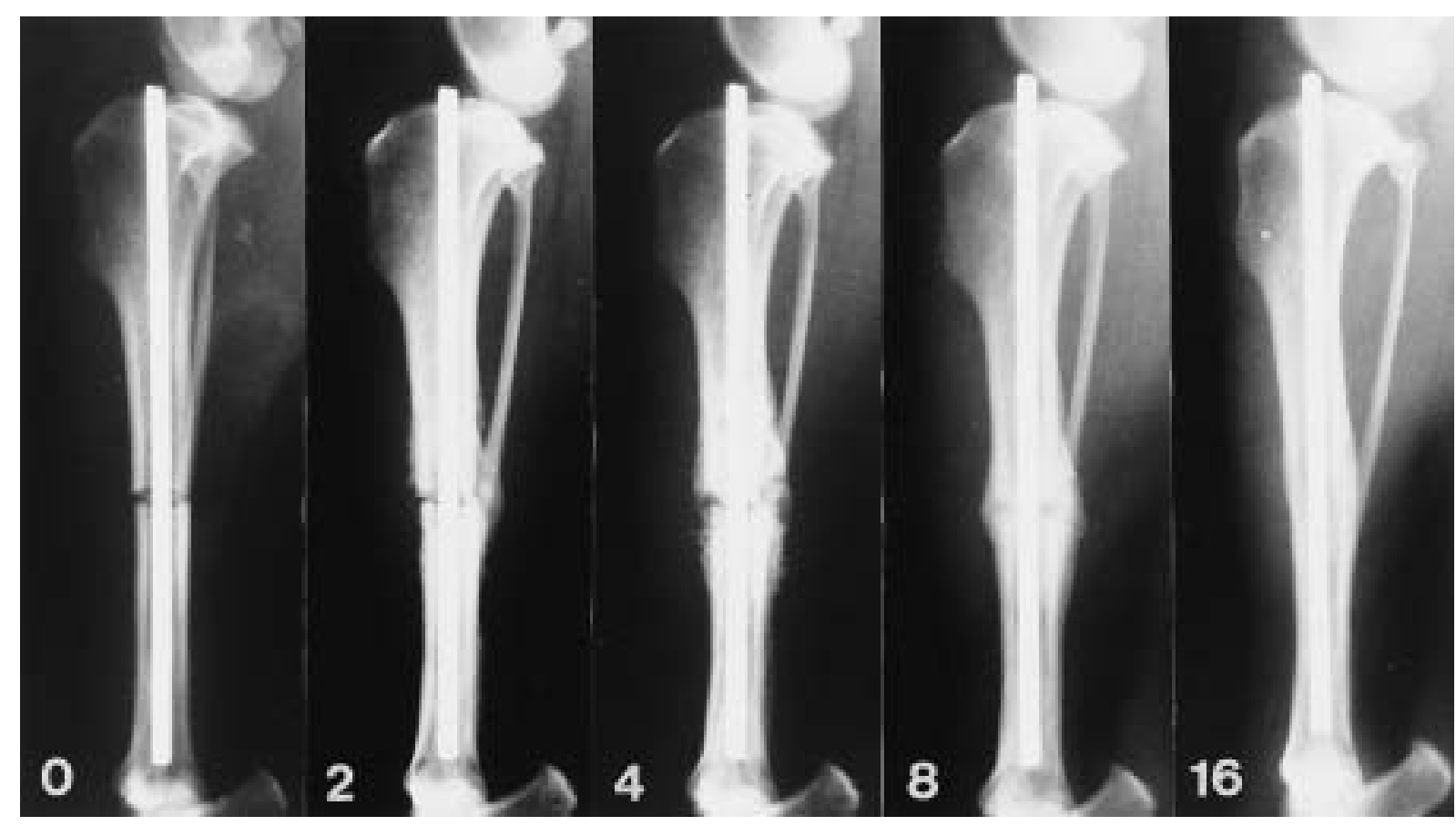

Fig. 1. Radiographic changes in the process of fracture healing. Each number represented the postoperative period in weeks. At 4 weeks, the periosteal callus was formed extensively near the osteotomized ends. At 8 weeks, the formation of external callus was comple ted and ossification at the osteotomized site became clearer. At 16 weeks, the remodeling of external callus was progressed, and the fracture line was completely disappeared. 


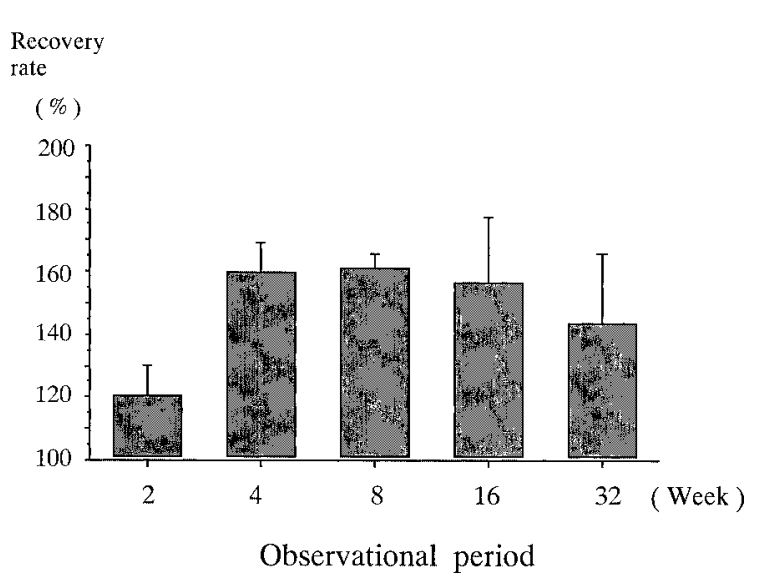

Fig. 2. Change of recovery rate of bone mineral content with time. $\mathrm{Y}$ axis represented the recovery rate of bone mineral content(BMC). The recovery rate means the ratios of BMC value on surgically treated side to that on the intact side. Each columun and each bar represented the mean value and the standard error, respectively.

Compared to ML, the recovery of MBS was relatively slower. The recovery rate of MBS ranged from 13.9 to $20.7 \%$ from 2 to 16 weeks. It was significantly increased to $76.1 \%$ at 32 weeks, compared to those at 2-16 weeks $(\mathrm{p}<0.001)$. The recovery rate of FR tended to increase with time, showing $87.0 \%$ in recovery rate at 32 weeks. Significant differences were found at 32 weeks compared to those at 2-16 weeks $(\mathrm{p}<0.01-\mathrm{p}<0.05)$. Compared to FR, the recovery of YM was relatively slower. The recovery rate of YM ranged from 6.3 to $12.8 \%$ from 2 until 16 weeks. It was significantly increased to $68.9 \%$ at 32 weeks, compared to those at $2-16$ weeks $(\mathrm{p}<0.001-\mathrm{p}<0.01)$.

In the field of small animal orthopedics, there has been a few quantitative informations on the mechanical parameters of the healing bone after fracture $[2,11,16]$. In the present study, we evaluated the changes in biomechanical characteristics of the healing bone after osteotomy during the healing process.

There were no significant differences in any findings for the mechanical parameters on the contralateral tibia between each group. Moreover, the mechanical parameters of the intact tibia measured in the present study are similar to that reported previously [16]. Two types of healing process, namely primary union and secondary union, have been known for the diaphyseal fracture of a long bone [7]. In the present study, healing was radiographically confirmed to proceed by the secondary union in all dogs. The average periods of anticipated healing time for the typical uncomplicated fracture treated in optimal fashion have been also reported [3, 13]. Taking into consideration on the ages of the animals and the method of fixation used in the present study, the radiographical findings at 16 weeks after surgery seemed to indicate that bone union was completed within the normal period in osteotomy models used in the present study [13]. Namely, the osteotomy line was disappeared, and the sufficient external callus was formed until 16 weeks on the sequential radiographs. Moreover, the recovery rate of BMC showed the tendency to increase from 4 to 16 weeks after surgery. It was suggested that these findings revealed progression of bone remodeling at the cortical region. However, on evaluating these changes of mechanical parameters comprehensively, it was found that all of the mechanical parameters measured failed to restore sufficient

Table 1. Changes in mechanical parameters in the healing process of the experimentallyinduced tibial fracture

\begin{tabular}{|c|c|c|c|c|c|c|c|}
\hline \multirow{2}{*}{\multicolumn{3}{|c|}{ Mechanical parameters }} & \multicolumn{5}{|c|}{ Observational period (Weeks) } \\
\hline & & & 2 & 4 & 8 & 16 & 32 \\
\hline \multirow{4}{*}{$\begin{array}{l}\text { Maximum load } \\
\text { ( N ) }\end{array}$} & Left & Mean & 1116.0 & 1143.3 & 1047.6 & 1243.8 & 1202.5 \\
\hline & & S.D. & 67.6 & 34.3 & 308.8 & 312.5 & 310.2 \\
\hline & Right & Mean & 290.9 & 401.5 & 404.8 & 588.3 & 1120.3 \\
\hline & & S.D. & 66.9 & 73.5 & 161.0 & 138.7 & 329.5 \\
\hline \multirow{4}{*}{$\begin{array}{l}\text { Maximum strength } \\
\qquad(\mathrm{MPa})\end{array}$} & Left & Mean & 209.5 & 219.0 & 233.7 & 277.0 & 289.8 \\
\hline & & S.D. & 29.9 & 10.9 & 10.0 & 79.5 & 34.0 \\
\hline & Right & Mean & 41.3 & 45.4 & 32.5 & 56.4 & 220.4 \\
\hline & & S.D. & 18.7 & 11.1 & 11.7 & 28.6 & 53.6 \\
\hline \multirow{4}{*}{$\begin{array}{l}\text { Frexural rigidity } \\
\quad(\mathrm{Nmm})\end{array}$} & Left & Mean & 1.9 & 1.8 & 2.4 & 2.2 & 2.3 \\
\hline & & S.D. & 0.5 & 0.3 & 0.9 & 0.4 & 0.8 \\
\hline & Right & Mean & 0.2 & 0.4 & 0.6 & 0.8 & 2.0 \\
\hline & & S.D. & 0.1 & 0.1 & 0.3 & 0.2 & 0.5 \\
\hline \multirow{4}{*}{$\begin{array}{l}\text { Young's modulus } \\
\text { (GPa) }\end{array}$} & Left & Mean & 4.8 & 3.9 & 8.0 & 7.3 & 7.4 \\
\hline & & S.D. & 1.3 & 1.4 & 2.4 & 2.7 & 4.2 \\
\hline & Right & Mean & 0.3 & 0.5 & 0.5 & 0.9 & 5.1 \\
\hline & & S.D. & 0.1 & 0.1 & 0.1 & 0.6 & 0.4 \\
\hline
\end{tabular}




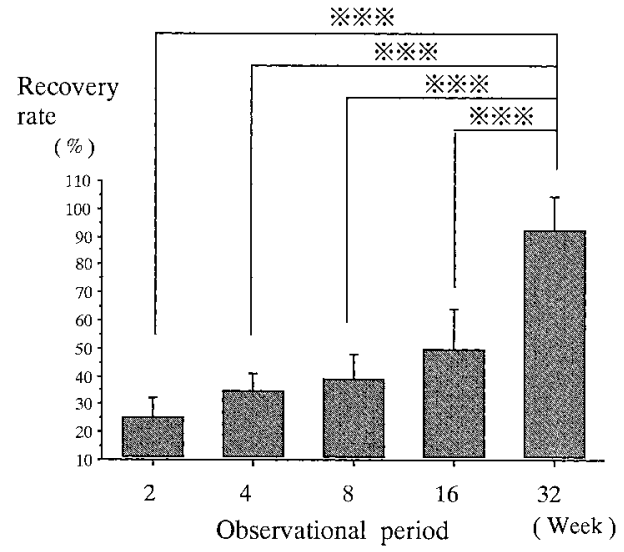

Maximum Load

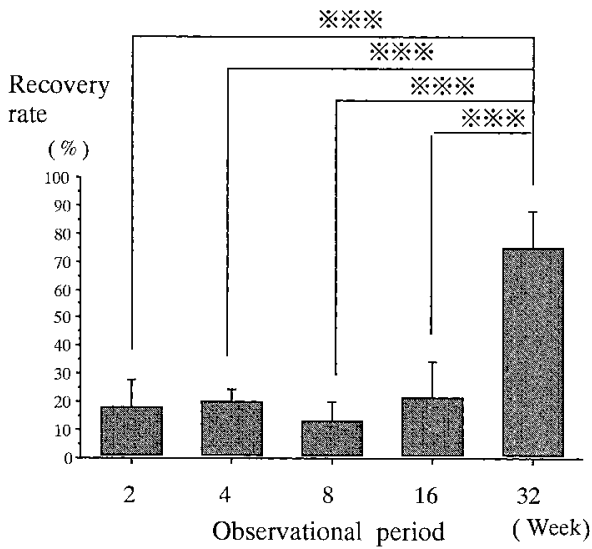

Maximum Bending Strength

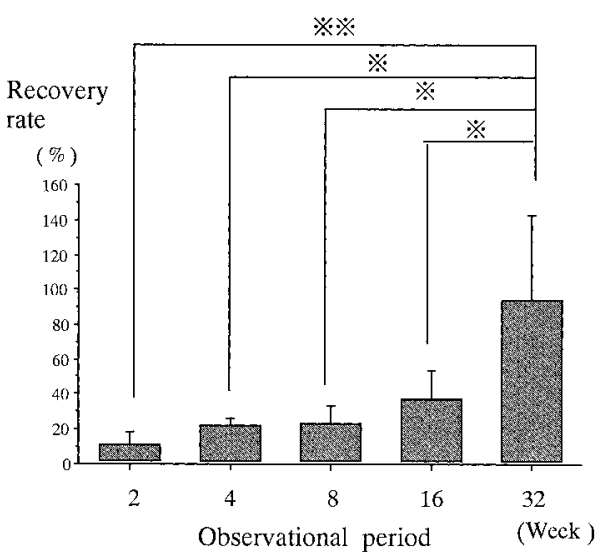

Frexural Rigidity

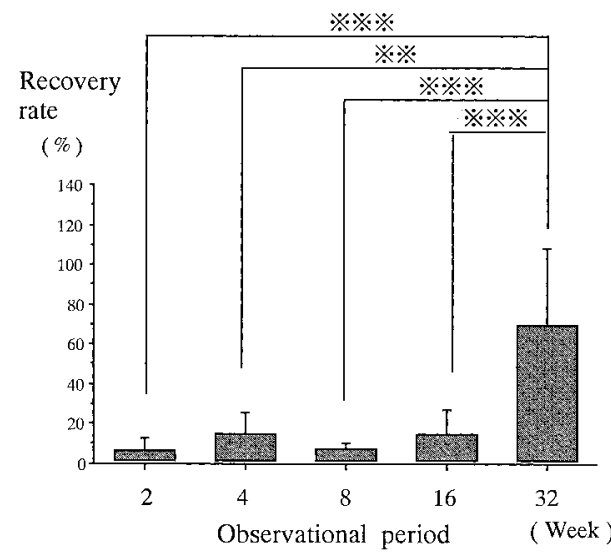

Young's Modulus

Fig. 3. Recovery of mechanical parameters of the fractured tibia with time. Y axis represented the recovery rate of each mechanical parameter. Recovery rates mean the ratios of measured value on surgically treated side to that on the intact side. Each columun and each bar represented the mean value and the

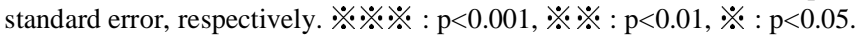

levels in 16 weeks. The recovery rates of them at 16 weeks were significantly lower than those at 32 weeks. Braden et $a l$. reported the mechanical parameters of the healing bone against torsional loads in their canine osteotomy model, in which the dogs were experimentally osteotomized and fixated by the intramedullary pinning technique [2]. They mentioned that the ultimate strength and torsional rigidities of the healing bone compared to the contralateral normal bone recovered 61.9 and $58.3 \%$, respectively, after 10 weeks [2]. In the present study, although we evaluated mechanical characteristics against bending loads of the tibia, the recovery rates of maximum load and flexural rigidity against bending loads showed 47.3 and $36.4 \%$, respectively, after 16 weeks.

On a point of view in blood supply to fractured bone, it has been known that intramedullary pin fixation causes less disruption to extraosseous blood supply than plating fixation, although insertion of any type of intramedullary pin disrupts the medullary vasculature, resulting in blockage of medullary afferent flow [8]. Biomechanically, it has also been known that the advantages of intramedullary pin fixation is their resistance to applied bending loads, compared to other fixation techniques, such as bone plating. On the contrary, biomechanical disadvantages of intramedullary pins include poor resistance to compressive or rotational loads and lack of fixation with bone [8]. In the field of orthopedics, it has been widely recognized that some situation in which the bone is protected from the mechanical stress, including the rigid plate fixation for fracture or immobilization of the limb, decrease the mechanical strength of the 
bone, and called as stress-shielding phenomenon $[5,9,11$, $12,17,18]$. In the present study, osteotomized area of the tibia had been protected from bending loads by the intramedullary pin during the observational period. Moreover, the intramedullary pin might interfere in medullary circulation. These might be the factors that delayed the recovery of bending strength. Therefore, removal of the intramedullary pin would be needed for the healing bone to gain the mechanical strength to normal level.

Recently, a lot of the growth factors, including basic fibroblast growth factor, bone morphogenic proteins, and transforming growth factor - $\beta$, for the skeletal system have been found, and some of them have been investigated for clinical use in the field of veterinary orthopedics $[4,10]$. It was also considered that evaluating the recovery of the mechanical parameters objectively using this osteotomy model would be suitable for the investigation on clinical efficacy of osteoinductive growth factors.

The results of the present study would indicate that the biomechanical characteristics of the healing bone could not recover sufficiently even after the passage of the healing period which has been empirically proposed from clinical findings. It was also suggested that the quantitative information concerning the mechanical strength of the healing bone after fractures should be clarified to reconfirm the healing process of the fracture, in the field of small animal orthopedics.

\section{REFERENCES}

1. Bak, B. and Jensen, K. S. 1992. Bone 13: 289-295.

2. Braden, T.D., Brinker, W.O., Little, R.W., Jenkins, R.B. and Butler, D. 1973. J. Am. Vet. Med. Assoc. 163: 65-69.
3. Brinker, W.O., Flo, G.L., Barden, T., Noser, G. and Merkley, D. 1975. J. Am. Anim. Hosp. Assoc. 11: 577- 586.

4. Canalis, E., McCarthy, T.L. and Centrella, M. 1989. J. Endocrinol. Invest. 12: 577-584.

5. Field, J.R. 1997. Vet. Comp. Orthop. Traumatol. 10: 88-94.

6. Grampp, S., Jergas, M., Glüer, C.C., Lang, P., Brastow, P. and Genant, H.K. 1993. Radiol. Clin. North Am. 1: 1133-1145.

7. Gray Brown, S. and Kramers, P. C. 1993. pp. 671-677. In: Disease Mechanisms in Small Animal Surgery, 2nd ed. (Joseph Bojrab, M. ed.), Lea \& Febiger, Philadelphia, U.S.A.

8. Hulse, D.A. and Johnson, A.L. 1997. pp. 705-766. In: Small Animal Surgery (Fossum, T.W. ed.), Mosby-Year Book, Inc., St. Louis, U.S.A.

9. Kaneps, A.J., Stover, S.M. and Lane, N.E. 1997. Bone 21: 419-423.

10. Mason, D.R. and Renberg, W.C. 2001. Compend. Contin. Educ. Prac. Vet. 23: 272-278.

11. Noser, G.A., Brinker, W.O., Little, R.W. and Lammerding, J.J. 1977. J. Am. Anim. Hosp. Assoc. 13: 559-561.

12. Paavolainen, P., Karaharju, E., Slätis, P., Ahonen, J. and Holmström, T. 1978. Clin. Orthop. Related. Res. 136: 287-293.

13. Piermattei, D.L. and Flo, G.L. 1997. pp. 24-146. In: Handbook of Small Animal Orthopedics and Fracture Repair, 3rd ed. (Piermattei, D.L. and Flo, G.L. eds.), W.B. Saunders Company, Philadelphia, U.S.A.

14. Schatzker, J., Meutstege, F. and Prieur, W.D. 1998. In: Manuals of Internal Fixation in Small Animals, 2nd ed. (Brinker, W.O., Olmstead, M.L., Summer-Smith, G., and Prieur, W.D. eds.), Springer, Berlin, Germany.

15. Turner, C.H. and Burr, D.B. 1993. Bone 14: 595-608

16. Tshamala, M., van Bree, H. and Mattheeuws, D. 1994. Vet. Comp. Orthop. Traumatol. 7: 25-30.

17. Uhthoff, H.K., Bardos, D.I. and Liskova-Kiar, M. 1981. J. Bone Joint Surg. 63-B: 427-434.

18. Uhthoff, H.K. and Dubuc, F.L. 1971. Clin. Orthop. Related. Res. 81: 165-170. 